\title{
Research on Surface Sampling for Determination of Pesticide Residues in Pome Fruit
}

\author{
Yunxia Luan ${ }^{1,2}$, Hua Ping ${ }^{1,2}$, Zhihong Ma ${ }^{1,2}$, and Ligang Pan $^{1,2}$ \\ ${ }^{1}$ Beijing Research Center for Agrifood Testing and Farmland Monitoring, Beijing, China \\ ${ }^{2}$ National Engineering Research Center for Information Technology in Agriculture, Beijing, China \\ luanyx@nercita.org.cn
}

\begin{abstract}
A surface sampling method for analysis of pesticide residues in pome fruit was studied by layer-by-layer scan from pericarp to kernel. Phoxim residues of apples, pears and papayas was determined by GC-MS, respectively, in core, different layers of pulp, peel ,as well as the whole fruit. Phoxim residue content was decreased when depth of the layer increased. It was inferred from experimental data the the appropriate depth of surface sampling is $2.5 \mathrm{~mm}, 2 \mathrm{~mm}$ and $3.5 \mathrm{~mm}$ in apples, pears and papayas. Although the ratio of the pesticide in pericarp and the total $(\mathrm{Cp} / \mathrm{Ct})$ ranged from 6.241 to 9.262 in apple, 4.254 to 5.069 in pears, and 4.39 to 5.037 in papayas in different groups, the ratio of $\mathrm{Cp} / \mathrm{Ct}$ and quality of specific group is essentially unchanged for a certain kind of fruit. According to the results, a conversion formula of the content of pesticide residues in whole fruit and pericarp was set, based on surface sampling method.
\end{abstract}

Keywords: Pome fruit, Pesticide residues, Phoxim, Surface sampling.

\section{Introduction}

One of the major roles of public health agencies is to ensure safe products for consumers through analysis of residual agricultural chemicals and veterinary drugs in foods. The use of agricultural chemicals and veterinary drugs in agriculture is necessary to improve the quality of the food produced. They play a beneficial role in providing a plentiful, low-cost supply of high-quality food. On the other hand, as a consequence of this use, the presence of residues in food that is a critical element of overall public health is unavoidable, and residues in food are of great importance in the evaluation of food quality. Organophosphorus (OP) pesticides are the most widely used agricultural pesticide. More than $70 \%$ of that are hyper-toxic or high-toxic, and normally are forbidden to use in planting fruits and vegetables. OP insecticides are routinely applied to fruit crops (e.g., apple, peach, pear) that are subject to consumption as single servings [1]. Consumption of individual fruits containing high OP insecticide residues may result in high exposure over a short period of time. Residue testing for the presence 
of pesticides, however, is routinely performed on composites of 5 to 10 individual or unit samples rather than samples of individual fruit [2].

Experimental study of the fruit for pesticide residues in agricultural products exist in the form, that is attached to the fruit into the fruit surface and the distribution of pesticides within the fruit. It is necessary to provide a representative sample to the laboratory for analysis [3]. No matter how carefully laboratory analyses are carried out, the results will be of limited value unless the sample provided is truly representative of the sample being tested [4]. The uneven distribution of pesticide residues among the treated objects leads to an inevitable variability of pesticide residue levels measured in the samples, which may significantly contribute to the combined uncertainty of the analytical results. Data generated in the UK have indicated that pesticide residue levels can be highly variable between the individual fruit from the same crop or lot in trade. Many fruit or vegetables in trade are mixed after harvest to form combined lots [5]. Analysis of composite samples provides a good indication of average residues but, where the lot has been mixed, such average values are potentially misleading [6]. Residues monitoring is the best means available for general control of pesticide use and consumer exposure, but new strategies for sampling and analysis are required to address the combined effects of residues variability and mixing of lots.

Development of analytical methods for pesticides in agri-food requires precise method of sampling. Pome fruit peel is the main remaining parts, parts of the peel residue is a measure of its ability to meet the safety standard limit fruit key factor. The apple pesticide residue question has become the chief obstacle in affecting the apple industry internationalization of our country [7]. At present, samples of pome fruit pesticide residue mainly from the entire apple or some edible parts, because the results from these have the generalization. However, the pesticide residue mainly exists in the pericarp. Therefore, the careful research to pesticide residue of the pericarp provides the theory instruction and the design basis to the pesticide residue and the elimination research.

However, limited data have been reported that directly compare the sampling procedures against one another. Even less information is published regarding the most appropriate part of the material to use in conjunction with a sampling procedure for pesticides detection, which is particularly important to the portable rapid detector of contamination in fruits and vegetables [8]. In our Previous studies, the temporal and spatial variations of phoxim residue in apples were studied. The results show that half-life of phoxim in apples was 1.64 days. The phoxim residue order in different position of apple is: pericarp >entire apple $>$ sarcocarp $>$ kernel and the ratio of residue in pericarp and the entire apple ranged from 6.241 to 9.262 in groups with different specific surface area [9]. The results indicate that, there are significant differences in the contents of residues between the pericarp and entire apple, which provide evidence in the theory instruction and design basis to the sampling method of pesticide residue based on pericarp. In this paper, further research was carried out on a surface sampling method for analysis of pesticide residues in pome fruit was studied by layer-by-layer scan from pericarp to kernel, and to explore the relationship between the content of pesticide in pericarp and the whole fruit. 


\section{Materials and Methods}

\subsection{Chemicals and Reagents}

Phoxim standard was purchased from National Institute of Metrology P.R.China. High-purity solvents cyclohexane, acetone, and acetonitrile were all purchased from Fisher Chemicals [10]. Analytical reagents magnesium sulfate and sodium chloride were purchased from Beijing Chemical Reagents Company (China). High-purity solvents acetonotrile, suitable for liquid chromatography, gas chromatography, and residue analysis used in the extraction and cleanup steps, were purchased from EMD Biosciences Inc(Mississauga, ON). Reagent grade $\mathrm{MgSO}_{4}$ and sodium chloride were also purchased from EMD Biosciences Inc.

\subsection{Apparatus}

The gel permeation chromatography (GPC) equipped with gas chromatography mass spectrometry system (GC/MS) was used. The GPC consists of two LC-10ADvp pumps, a SIL-10ADvp auto-sampler, a Shodex CLNpak EV-200AC column $(2 \mathrm{~mm}$ i.d. $\times 150$ $\mathrm{mm}$ ) and CTO-10ASvp column oven, a SPD-10Avp UV detector, two FCV-12AH flow channel selectionvalves (RV.A, RV.B) and a SCL 10Avp system controller. GC/MS machine is a Shimadzu GC/MS-QP2010 instrumentation equipped with a PTV-2010 large-volume injection device.

GC/MS data analysis was triggered by a contact closure start signal from the HPLC controller. Data acquisition was performed using a C-R8A plus data processor. All these parts are the products of Shimadzu (Kyoto, Japan), except the Shodex CLNpak EV-200AC column (Shoko Co., Tokyo, Japan). Acetone/ cyclohexane mixing solvent $(3 / 7, v / v)$ was used as the mobile phase of GPC [11], and the flow rate was set at 0.1 $\mathrm{mL} / \mathrm{min}$. The mobile phase was degassed using DGU-14A degasser (Shimadzu), and the GPC column was kept at $40^{\circ} \mathrm{C}$ in the column oven.

\subsection{Sample Preparation}

All samples were purchased at local markets in Beijing. Samples were extensively collected to achieve good sample homogeneity. The phoxim compounds were applied using an air blast sprayer and application rates guaranteed to contain $50 \%$ phoxim emulsifiable with the dilution multiple of 1:500. Individual fruits were cored and sliced into 5 segments (pericarp(P), sarcocarp1(S1), S2,S3,S4 and kernel) using a corer/slicer retailed and peeling machine for domestic use. The first and alternate slices of each apple were taken, chopped manually using a knife, and placed in a plastic bag for storage at $-25^{\circ} \mathrm{C}$ until extraction and analysis. The remaining segments of individual apples were retained in separate bags for preparation of composite samples. Composites were constructed from the apples prepared from each group by randomly selecting 8 apples for each treat, without regard to the style and weight of each apple. Composite samples were prepared by thoroughly mixing the chopped apple pieces 
from the bags containing the retained portions of the individual apples. Composite samples were frozen until extraction and analysis. To prepare each sample, $10 \mathrm{~g}$ of a previously homogenized food materialwas transferred into a suitable glass vessel. Then, $10 \mathrm{~mL}$ acetonitrile was added to each sample using an adjustable-volume solvent dispenser. The glass vessels were capped before vortex mixing for $1 \mathrm{~min}$ at maximum speed. Once the initial sample mixing was completed, $1 \mathrm{~g} \mathrm{NaCl}$ and $4 \mathrm{~g}$ anhydrous $\mathrm{MgSO}_{4}$ were added and mixed immediately on a Vortex mixer for $1 \mathrm{~min}$. It is important to note that this step must be taken immediately after the initial mixing step to prevent the formation of $\mathrm{MgSO}_{4}$ conglomerates. To separate phases, samples were centrifuged for $10 \mathrm{~min}$ at $1570 \times \mathrm{g}$. Using an adjustable repeating pipette, $1.0 \mathrm{~mL}$ aliquot of upper acetonitrile layer was transferred into a $1.5 \mathrm{~mL}$ flip-top microcentrifuge vial containing $150 \mathrm{mg}$ anhydrous $\mathrm{MgSO}_{4}$ and $50 \mathrm{mg}$ PSA sorbent. The vial was tightly capped and shaken on a vortex mixer for 1 min before extraction. Then the mixed extraction solution was centrifuged for $5 \mathrm{~min}$ to separate solids from solution. The solution was then transferred into an autosampler for GPC-GC/MS analysis.

\subsection{Determination of Phoxim in Apple by GPC-GC/MS}

Analysis was performed by GPC-GC/MS. The analytical GC/MS equipped with a PTV-2010 large-volume injection device was carried out using deactivated silica tubing $(5 \mathrm{~m} \times 0.53 \mathrm{~mm}$ i.d. $)$, a Rtx-5MS pre-column $(5 \mathrm{~m} \times 0.25 \mathrm{~mm})$ and a Rtx-5MS column $(30 \mathrm{~m} \times 0.25 \mathrm{~mm} \times 0.25 \mu \mathrm{m}$ film thickness; coated with $5 \%$ phenyl and $95 \%$ methylpolysiloxane; Restek Corporation, Bellefonte, Palo Alto, USA). The temperature of the PTV injector was set at $120^{\circ} \mathrm{C}$ for the initial $5 \mathrm{~min}$ of sampling time, and then increased to $250^{\circ} \mathrm{C}$ at $100^{\circ} \mathrm{C} / \mathrm{min}$. The oven temperature was maintained at $80^{\circ} \mathrm{C}$ for $5 \mathrm{~min}$, subsequently increased to $280^{\circ} \mathrm{C}$ at a rate of $8^{\circ} \mathrm{C} / \mathrm{min}$, and then held constant for $10 \mathrm{~min}$ [12]. The quadrupole mass spectrometer was operated in the electron impact ion (EI) mode. Ion source temperature and interface temperature were set at $200^{\circ} \mathrm{C}$ and $250^{\circ} \mathrm{C}$, respectively. The mass spectrometer was operated in an ionizing energy of $70 \mathrm{eV}$. Injection volumes were $1 \mu \mathrm{L}$ for all analyses. Helium $(99.999 \%)$ was used as the carrier gas [13].

\subsection{Quality Assurance/Quality Control}

Apples from market with no phoxim insecticide application were obtained, prepared the same as the rinsed and peeled apples, and were used to prepare blank apple matrix for quality assurance testing. With each set of samples 10-15 samples per set) extracted and analyzed, two aliquots of blank apple matrix were prepared for separate extraction followed by extraction, cleanup, and analysis as for all other samples. Background levels of some analysis were periodically detected in the blank matrix samples and were used for background subtraction in the determination of recovery from spiked matrix only; residue concentrations in samples were not blank corrected. No traces of the phoxim were observed in any of the reagent blanks analyzed. 


\section{$3 \quad$ Results and Discussion}

\subsection{Determine of the Parameters for Surface Sampling}

In order to determine the exact thickness of pericarp and sampling style, the materail was cut by $1.5 \mathrm{~mm}-2 \mathrm{~mm}$ each piece from the pericarp to kernel, and pesticide residues in fruit peel, kernel and pulp of different depth were determined. Taking the Fuji apple as example, according to dilution ratio of 1:1000 dilution of Phoxim EC, more than fifteen days after spraying pesticide on fruit, pesticide residues in samples was detected according to the method of NY/T 761.1-2004 "vegetables and fruits organophosphorus, organochlorine, pyrethroid and carbamate pesticide multi-residue methods-Part 1 of fruits and vegetables organophosphorus pesticide multi-residue method" and GB/T5009.199-2003 "vegetables of organophosphorus and carbamate pesticide residues in rapid detection." for lab and rapid detection. Each sub-sample was taken from 15 samples of different shape and weight. The results are presented in Figure 1.

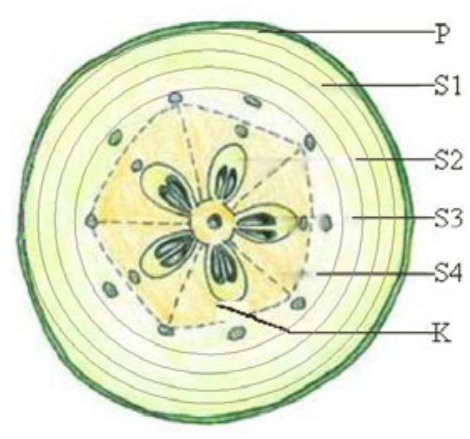

A

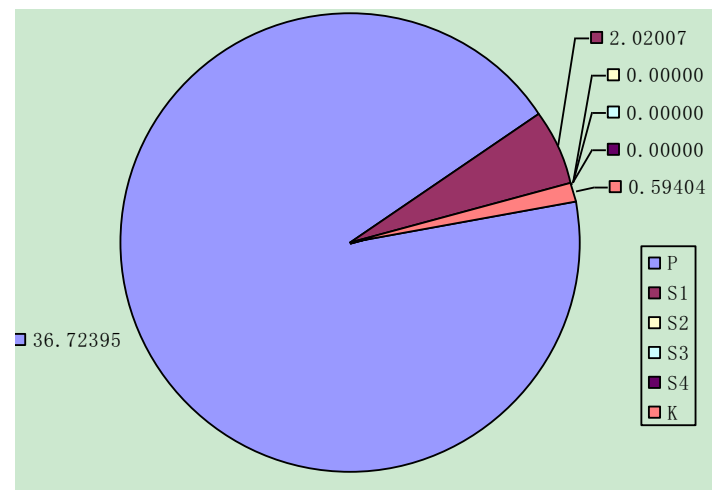

B

Fig. 1. Compartmentalization and sampling methord of apple (A) and the content of Phoxim in each depth (B)

$\mathrm{P}$ : pericarp of apples with $0.5 \mathrm{~mm}$ pulp, S1: The first layer with $2 \mathrm{~mm}$ pulp, S2: the second layer with $2 \mathrm{~mm}$ sarcocarp, S3: the third layer with $2 \mathrm{~mm}$ sarcocarp, S4: the sarcocarp near kernel, $\mathrm{K}$ : kernel or core of apples.

Experimental study of the pesticide residues in pome fruit, which is attached to the fruit surface and the distribution of pesticides within the fruit was carried out. From experiments performed and analysis of pesticide residues in different layer from pericarp to kernel, it is showed that, pesticide was detected maily in layer P and S1, the pesticide in pericarp and the first layer with $2 \mathrm{~mm}$ pulp account for $98.49 \%$ of the whole sample. Through different parts of the fruit residue test results revealed that the major part of remaining in the apple peel, fruit pulp and other parts of the residual is minimal. According to the apple skin to the stone from the stratified analysis, pesticide residues, mainly in the skin and pulp of the following $2.5 \mathrm{~mm}$, this part of the sample selected sampling to detect the levels of residues of pesticides, because this part of the high levels of pesticide residues, can increase rapidly screening test accuracy. 
According to the experimental results, the standard design of the skin sampling methods, reduction in access to sub-samples the following $2.5 \mathrm{~mm}$ thick skin and flesh as the test object.

\subsection{Study of the Weight Coefficient of Pesticide Residues in Pericarp}

Selected based on the standard higher levels of pesticide residues in samples of skin as a target for different types of fruits, pesticide residues on its surface and overall there is a certain amount of pesticide residue conversion relationship [14]. The research was supported by the skin of pesticide content in the trend over time to determine the appropriate sampling time interval after application, and in accordance with the standard parameters of the specific sampling techniques to obtain test samples, the research team through the following experiments, made pome fruit surface pesticide residue content and the overall coefficient of pesticide content in the skin pesticide residues.

Using Apple, pear, and papaya $(n=15)$, sampling the surface homogenized pericarp(with $2.5 \mathrm{~mm}$ pulp) as detection material, homogenized with a whole apple parallel processing [15], determination of pesticide content, access to pesticide skin factor, diluted with water according to the proportion of 1:1000 dilution phoxim EC, using the method of spraying pesticide on apples [16]. According to NY/T761.1-2004 "vegetables and fruits organophosphorus, organochlorine, pyrethroid and carbamate pesticide multi-residue methods-Part 1 of fruits and vegetables organophosphorus pesticide multi-residue method" approach with gas chromatography of whole fruit and pericarp of the accurate detection of pesticide residues pesticide residues in order to obtain skin factor [17].

As the result in Fig2-4, the three kinds of fruit showed various levels of pesticide in pericarp and the whole fruit, though the content in pericarp was much higher than the total homogenate. So, the ratio of the pesticide in pericarp and the whole fruit $(\mathrm{Cp} / \mathrm{Ct})$ was got from the three kinds of fruits with different shape and quality as shown in Table1.

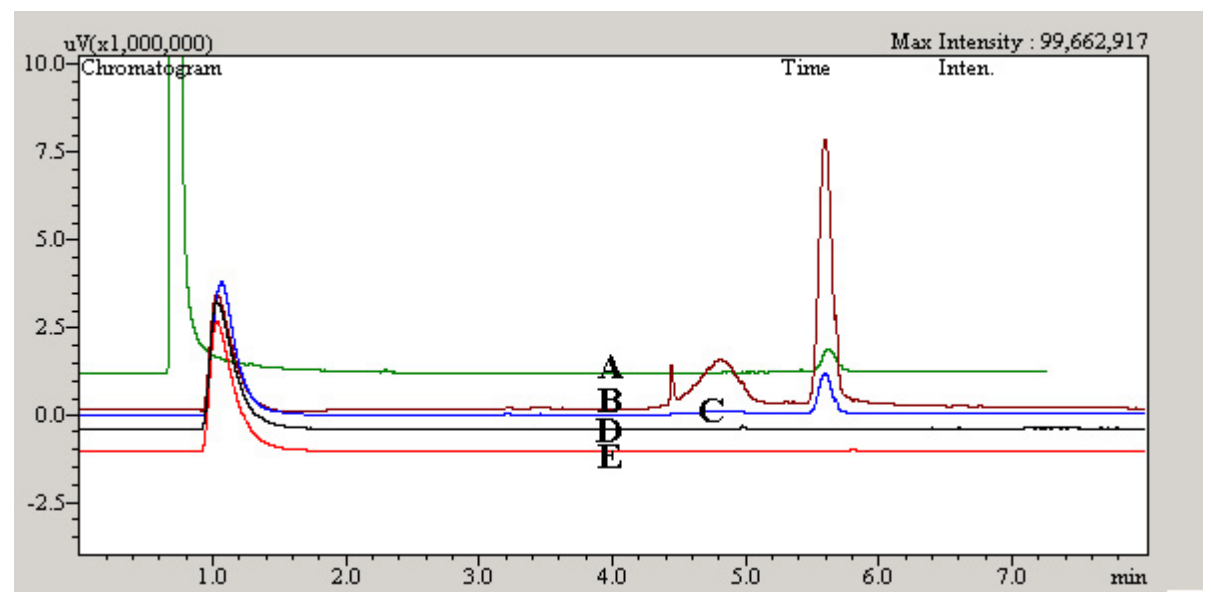

Fig. 2. GC diagram of phoxim in Fuji apple

A phoxim $5.25 \mathrm{mg} / \mathrm{kg}$; B sample of pericarp;C sample of the whole fruit; D and E as CK. 


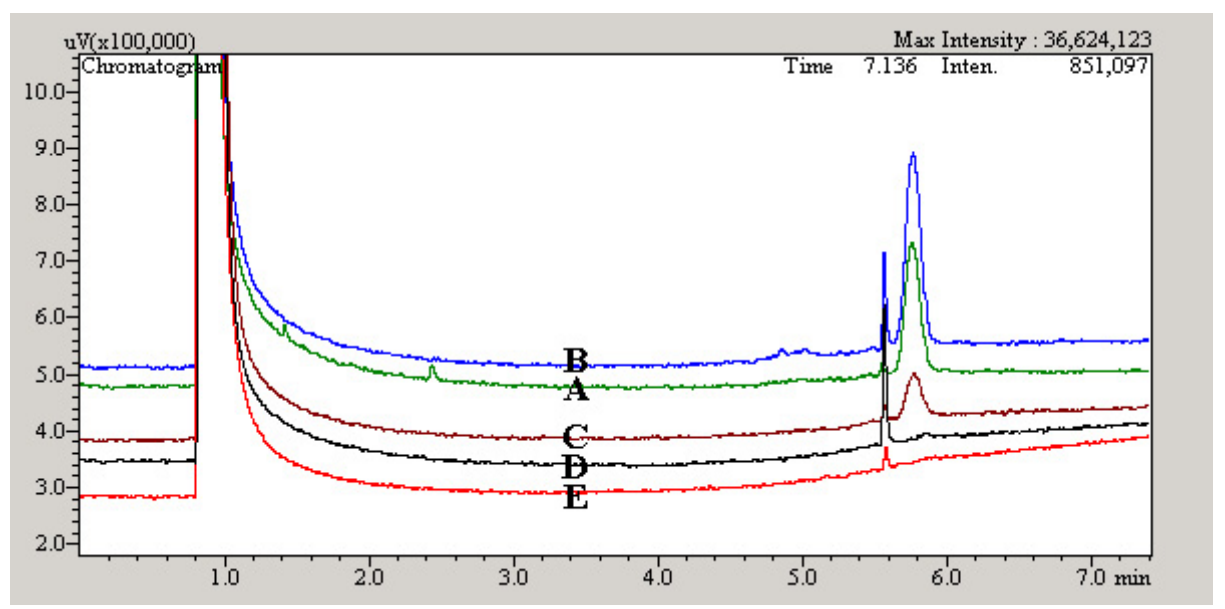

A phoxim $5.25 \mathrm{mg} / \mathrm{kg}$; B sample of pericarp;C sample of the whole fruit; D and E as CK.

Fig. 3. GC diagram of phoxim in pear

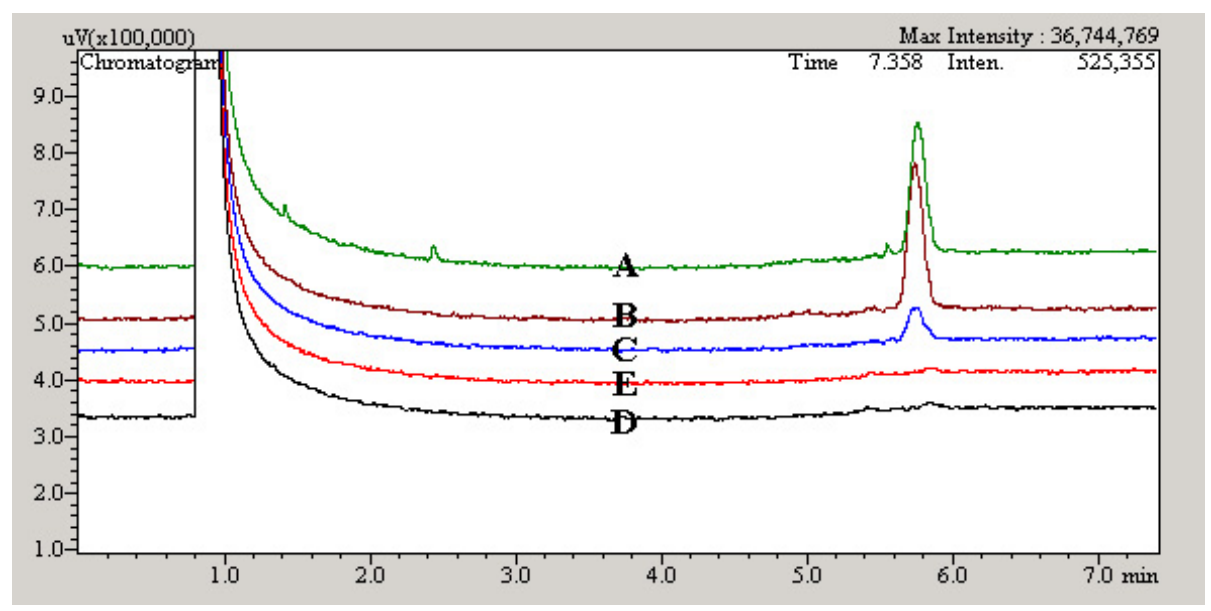

A phoxim $5.25 \mathrm{mg} / \mathrm{kg}$; B sample of pericarp;C sample of the whole fruit; D and E as CK.

Fig. 4. GC diagram of phoxim in papaya 
Table 1. Weight Coefficient of pesticide residues in pericarp of common pome fruit

\begin{tabular}{|lcc|}
\hline Fruit & Quality $(\mathbf{g})$ & $\mathbf{C}_{\mathbf{p}} / \mathbf{C}_{\mathbf{T}}$ \\
\hline Fuji apple & $169.605 \pm 4.666$ & 6.241 \\
& $189.425 \pm 4.748$ & 7.963 \\
& $209.032 \pm 4.015$ & 9.262 \\
Pear & $135.098 \pm 3.411$ & 4.254 \\
& $146.270 \pm 3.566$ & 4.516 \\
& $159.215 \pm 3.652$ & 5.069 \\
Papaya & $1060.96 \pm 10.21$ & 4.390 \\
& $1072.21 \pm 10.33$ & 4.815 \\
& $1080.67 \pm 11.19$ & 5.037 \\
\hline
\end{tabular}

\subsection{Formula for Calculating the Amount of Pesticide Residues in the Epidermis}

Although the ratio of the pesticide in pericarp and the total $(\mathrm{Cp} / \mathrm{Ct})$ ranged from 6.241 to 9.262 in apple, 4.254 to 5.069 in pears, and 4.39 to 5.037 in papayas in different groups of weight, the ratio of $\mathrm{Cp} / \mathrm{Ct}$ and quality of specific group is essentially unchanged for a certain kind of fruit (Table 2). According to these results, a conversion formula of the content of pesticide residues in whole fruit and pericarp was set based on surface sampling method.

The weight of a pesticide(a) refers to the epidermis and fruit pesticide content ratio of the overall pesticide content(1).

$$
a=\frac{C_{p}}{c_{t}}
$$

$a$ : Weight coefficient of pesticide residues in pericarp,

$C_{t}$ : total pesticide content,

$C_{p}$ : pesticide content in the epidermis

Coefficient A refers to the contribution of the content of epidermal pesticide to the whole fruit

$$
A=\frac{a}{m}
$$

A: surface pesticide coefficient,

a: Weight coefficient of pesticide residues in pericarp,

$m$ : the quality of a single sample

According to the data of Weight coefficient of pesticide residues in pericarp of common pome fruits in Table1, the surface pesticide coefficient could be calculated 
based on formula (2). As shown in Table2, the surface pesticide coefficient A of Fuji apple, pear and papaya were $0.041,0.031$ and 0.004 respectively.

So fruit samples with pesticide residues in total mass fraction of $w$, in units of milligrams per kilogram ( $\mathrm{mg} / \mathrm{kg}$ ), according to the measured pesticide residues in skin samples according to the formula (3)

$$
w=\frac{w_{p}}{A \times m}
$$

$w$ - the total pesticide residues in fruits, in milligrams per kilogram $(\mathrm{mg} / \mathrm{kg})$;

$\mathrm{w}_{\mathrm{p}}$ - the amount of pesticide residues in fruit pericarp, in milligrams per kilogram $(\mathrm{mg} / \mathrm{kg})$

$m$ - single sample sample quality of the sample, in grams $(\mathrm{g})$;

A - surface pesticide coefficient

Table 2. Surface pesticide coefficient in common pome fruit

\begin{tabular}{cc}
\hline Fruit & $\mathrm{A}$ \\
\hline Fuji apple & 0.041 \\
Pear & 0.031 \\
Papaya & 0.004 \\
\hline
\end{tabular}

\subsection{Application of the Surface Sampling Method on Rapid Detector of Pesticides}

Select the most commonly used Rapid detector of Pesticides or detector have a fast detector function, to detect pesticides in food safety rapid detector to verify the sampling method, the results shown in Table 3. Take apples, pears and papaya samples of different quality and shape of the three groups of 15 individuals, according to this standard involve the rapid detection of pesticide residues in fruit skin sampling methods in the technical parameters, take the skin (with $2.5 \mathrm{~mm}$ pulp) as sample, while the overall homogenized fruit sample, according to GB/T 5009.199-2003 "vegetables of organophosphorus and carbamate pesticide residues in the rapid detection," Determination of pesticide content. It can be seen from the results, obtained in accordance with the samples of fruit skin test results of pesticide residues, pesticide residues on the enzyme that the inhibition rate was significantly greater than the overall sample of inhibition of fruit (see Table 3).

The result of rapid detectors using whole apple sample and surface sample demonstrated that, a surface sampling method for analysis of pesticide residues in pome fruit could increased sensitivity and reduced the average impact of whole fruit homogenates. Combination of the phoxim residues of apples, pears and papayas determined by GC-MS in different layers of the fruit and the formula for calculating the amount of pesticide residues in the epidermis amount of pesticide residues in the epidermis, an efficient surface sampling method was studied and established. 
Table 3. Comparision of the results of rapid detectors using different sampling method

\begin{tabular}{cc}
\hline Sample & Inhibition rate $(\%)$ \\
\hline Whole apple & $34.6 \pm 5.31$ \\
Pee1 of apple & $84.4 \pm 4.89$ \\
Whole pear & $30.1 \pm 5.83$ \\
Peel of pear & $71.8 \pm 3.26$ \\
Whole papaya & $35.4 \pm 2.91$ \\
Pee1 of papaya & $91.3 \pm 6.03$ \\
\hline
\end{tabular}

\section{Discussion}

According to the reseach of pesticide residues in pome fruit by layer-by-layer scan from pericarp to kernel, the previous solution of the significant differences in the contents of residues between the pericarp and entire apple was demonstrated, further more these research revealed the pesticide residues, mainly remained in the pericarp and pulp of the following $2.5 \mathrm{~mm}$ thick, this part of the sample could be selected as sample to detect the levels of residues of pesticides, because this part of the high levels of pesticide residues, can increase rapidly screening test accuracy [18]. From the experimental results obtained, the standard design of the skin sampling methods, reduction in access to sub-samples the following $2.5 \mathrm{~mm}$ thick skin and flesh as the test object.

Scientific and standardized sampling method can reduce the skin samples and pulp composition on the average of the impact test results, reduce false positives and improve accuracy [19]. But different types of fruit, types of pesticides and interval time after spraying should be further studied to verify the coefficient and formula. Effect of surface waxes on the persistence of pesticide in Fuji apples, pears, and papayas would effect the diffusion of pesticide to pulp.

Coefficient A refers to the epidermal skin pesticide pesticide content of the fruit content of the overall contribution of major factors affecting A include the following factors: (1) differences include different types of fruit and fruit surface area for adsorption of pesticides in different penetrate the skin of the fruit impact of pesticides [20]. (2) determination of pesticide residues in skin samples when differences in sampling methods must be standardized to ensure that the skin sample, and with the standard method for sampling the epidermis consistent with the provisions. (3) the time factor after the recommended interval of sampling.

Therefore, this surface sampling method and the corresponding formula can be applied to various methods of detection of pesticide residues, in particular, the portable rapid detection machines. As the rapid development of materials science, biology and chemistry technologies, the surface sampling method would be widely used in sensor and other equipments. 
Acknowledgements. We thank the Special Fund for Agro-scientific Research in the Public Interest, the Ministry of Agriculture of the People's Republic of China (Project 201003008 and 201203046) and National Key Technology R\&D Program (Project 2009BADB9B07), for financial support.

\section{References}

1. Hamers, T., van den Brink, P.J., et al.: Estrogenic and esterase-inhibiting potency in rainwater in relation to pesticide concentrations, sampling season and location. Environ. Pollut. 123(1), 47-65 (2003)

2. Fenske, R.A., Bradman, A., et al.: Lessons learned for the assessment of children's pesticide exposure: critical sampling and analytical issues for future studies. Environ. Health Perspect. 113(10), 1455-1462 (2005)

3. Wang, J., Tuduri, L., et al.: Flexibility of solid-phase microextraction for passive sampling of atmospheric pesticides. J. Chromatogr. A 1216(15), 3031-3037 (2009)

4. Wang, K., Shipp, J.L.: Sequential sampling plans for western flower thrips (Thysanoptera: Thripidae) on greenhouse cucumbers. J. Econ. Entomol. 94(2), 579-585 (2001)

5. Ambrus, A.: Estimation of sampling uncertainty for determination of pesticide residues in plant commodities. J. Environ. Sci. Health B 44(7), 627-639 (2009); Chromatogr A 1216(15), 2972-2983

6. Yusa, V., Coscolla, C., et al.: Sampling and analysis of pesticides in ambient air. J. Chemosphere 51(6), 509-513 (2009)

7. Neumann, M., Liess, M., et al.: A qualitative sampling method for monitoring water quality in temporary channels or point sources and its application to pesticide contamination (2003)

8. Shen, J., Liu, J.: Determination of carbendazim residue in orange and soil using high performance liquid chromatography. Se Pu. 27(3), 308-312 (2009)

9. Luan, Y., Ping, H., Pan, L.: Residue dynamics of phoxim in pericarp, sarcocarp and kernel of apple. In: Li, D., Liu, Y., Chen, Y. (eds.) CCTA 2010, Part II. IFIP AICT, vol. 345, pp. 457-464. Springer, Heidelberg (2011)

10. Shen, J., Liu, J.: Determination of carbendazim residue in orange and soil using high performance liquid chromatography. Se Pu. 27(3), 308-312 (2009)

11. Schafer, R.B., Paschke, A., et al.: Performance of the Chemcatcher passive sampler when used to monitor 10 polar and semi-polar pesticides in 16 Central European streams, and comparison with two other sampling methods. Water Res. 42(10-11), 2707-2717 (2008)

12. Schafer, R.B., Paschke, A., et al.: Performance of the Chemcatcher passive sampler when used to monitor 10 polar and semi-polar pesticides in 16 Central European streams, and comparison with two other sampling methods. Water Res. 42(10-11), 2707-2717 (2008)

13. Schafer, R.B., Pettigrove, V., et al.: Effects of Pesticides Monitored with Three Sampling Methods in 24 Sites on Macroinvertebrates and Microorganisms. Environ. Sci. Technol.

14. Bernard, C.E., Berry, M.R., et al.: Sampling household surfaces for pesticide residues: comparison between a press sampler and solvent-moistened wipes. Sci. Total Environ. 389(2-3), 514-521 (2008)

15. Rosenheck, L., Cowell, J., et al.: Determination of a standardized sampling technique for pesticide transferable turf residues. Bull. Environ. Contam. Toxicol. 67(6), 780-786 (2001)

16. Teng, H., Kwigizile, V., et al.: Investigation of the AP-42 sampling method. J. Air Waste Manag. Assoc. 58(11), 1422-1433 (2008) 
17. Li, H., Vermeirssen, E.L., et al.: Controlled field evaluation of water flow rate effects on sampling polar organic compounds using polar organic chemical integrative samplers. Environ. Toxicol. Chem. 29(11), 2461-2469

18. Rabiet, M., Margoum, C., et al.: Assessing pesticide concentrations and fluxes in the stream of a small vineyard catchment-effect of sampling frequency. Environ. Pollut. 158(3), 737-748

19. Berger, B., von Holst, C.: Pesticide residues in products of plant origin in the European Union. Sampling strategy and results from the co-ordinated EU monitoring programmes in 1996 and 1997. Environ. Sci. Pollut. Res. Int. 8(2), 109-112 (2001)

20. Gaultier, J., Farenhorst, A., et al.: Regional assessment of herbicide sorption and degradation in two sampling years. J. Environ. Qual. 37(5), 1825-1836 (2008) 